\title{
Mapping Implied Body Actions in the Human Motor System
}

\author{
Cosimo Urgesi, ${ }^{1,2}$ Valentina Moro, ${ }^{3}$ Matteo Candidi, ${ }^{4,5}$ and Salvatore M. Aglioti ${ }^{4,5}$ \\ ${ }^{1}$ Dipartimento di Scienze Neurologiche e della Visione, Sezione di Fisiologia Umana, Università di Verona, I-37134 Verona, Italy, ${ }^{2}$ Istituto di Ricovero e \\ Cura a Carattere Scientifico "E. Medea," Polo Regionale Friuli Venezia Giulia, I-33078 San Vito al Tagliamento, Pordenone, Italy, ${ }^{3}$ Dipartimento di \\ Psicologia e Antropologia Culturale, Università di Verona, I-37122 Verona, Italy, and 4Dipartimento di Psicologia, Università di Roma "La Sapienza," I- \\ 00185 Roma, Italy and ${ }^{5}$ Istituto di Ricovero e Cura a Carattere Scientifico Fondazione Santa Lucia, I-00179 Roma, Italy
}

The human visual system is highly tuned to perceive actual motion as well as to extrapolate dynamic information from static pictures of objects or creatures captured in the middle of motion. Processing of implied motion activates higher-order visual areas that are also involved in processing biological motion. Imagery and observation of actual movements performed by others engenders selective activation of motor and premotor areas that are part of a mirror-neuron system matching action observation and execution. By using single-pulse transcranial magnetic stimulation, we found that the mere observation of static snapshots of hands suggesting a pincer grip action induced an increase in corticospinal excitability as compared with observation of resting, relaxed hands, or hands suggesting a completed action. This facilitatory effect was specific for the muscle that would be activated during actual execution of the observed action. We found no changes in responsiveness of the tested muscles during observation of nonbiological entities with (e.g., waterfalls) or without (e.g., icefalls) implied motion. Thus, extrapolation of motion information concerning human actions induced a selective activation of the motor system. This indicates that overlapping motor regions are engaged in the visual analysis of physical and implied body actions. The absence of motor evoked potential modulation during observation of end posture stimuli may indicate that the observationexecution matching system is preferentially activated by implied, ongoing but not yet completed actions.

Key words: implied motion; motor cortex; transcranial magnetic stimulation; mirror neurons; body actions; representational momentum

\section{Introduction}

Humans are inherently able to detect and recognize the motion of visual stimuli, even when actual motion is not present but only implied. The term implied motion refers to dynamic information extracted from static images. Psychophysical studies have shown that still images implying motion are stored in memory as if the portrayed object or living being were indeed moving. Specifically, recognition memory of still images suggesting motion is distorted forward in the direction of the implied motion. This effect, termed representational momentum (Freyd, 1983), has been demonstrated with a variety of stimuli including dot patterns (Finke and Freyd, 1985), common objects (Finke and Shyi, 1988), and human figures (Verfaillie and Daems, 2002).

Neuroimaging studies in humans indicate that the medial temporal/medial superior temporal cortex (MT/MST complex) is typically activated during the visual experience of real or illusory motion, whether biological or nonbiological (Zeki et al., 1991; Dupont et al., 1994; Tootell et al., 1995). These areas are also involved in the processing of implied motion because they are more activated by photographs of humans, animals, and natural scenes with implied motion than by photographs devoid of

Received Jan. 20, 2006; revised May 5, 2006; accepted June 20, 2006.

This work was supported by grants from Ministero Italiano dell'Università e della Ricerca and Finanziamento Italiano per la Ricerca di Base, Italy. We thank Prof. Giovanni Berlucchi for his invaluable help in discussing the results.

Correspondence should be addressed to Salvatore M. Aglioti, Dipartimento di Psicologia, Università di Roma "La Sapienza," Via dei Marsi 78, I-00185 Roma, Italy. E-mail: salvatoremaria.aglioti@uniroma1.it.

D0I:10.1523/JNEUROSCI.1289-06.2006

Copyright $\odot 2006$ Society for Neuroscience $\quad 0270-6474 / 06 / 267942-08 \$ 15.00 / 0$ implied motion (Kourtzi and Kanwisher, 2000; Senior et al., 2000). Single neurons in the monkey superior temporal cortex respond to the presentation of both moving body parts and static images of body postures implying a preceding action (Jellema and Perrett, 2003; Puce and Perrett, 2003). Moreover, still images implying biological motion can activate the superior temporal sulcus (Peuskens et al., 2005) as well as parietal areas (Hermsdörfer et al., 2001), and the observation of static postures suggesting a transition to action selectively activates the lateral occipitotemporal junction (Peigneux et al., 2000). Thus, all of the studies performed so far suggest that implied motion processing activates only visual areas in the posterior cerebral cortex.

Although specific regions in the human superior temporal sulcus are activated by the visual processing of actual movements of human bodies and body parts (Grossman et al., 2000), neurophysiologic and neuroimaging studies in nonhuman and human primates show that viewing actual body movements engages a higher-order, mainly frontoparietal, network that is highly suitable for matching action observation with action execution and is referred to as the motor mirror system (for review, see Rizzolatti and Craighero, 2004). For example, single-pulse transcranial magnetic stimulation (TMS) studies show that action observation triggers specific mirror activation of the muscles involved in the actual execution of the very same action (for review, see Fadiga et al., 2005). Although movement information can be extracted or inferred from static images, so far there have been no investigations of the possible mapping of snapshots implying biological or nonbiological movements in the motor system. Here, we used single-pulse TMS to determine whether the observation 
of snapshots of hand postures stimuli, which may or may not imply motion, can engender a selective mirror facilitation of corticospinal motor representations. Moreover, we tested whether the motor mirror mapping of implied actions is based on the somatotopic rules used for mapping real actions.

\section{Materials and Methods}

Participants. Sixteen healthy individuals (eight women) aged 20-29 (mean, 23 years) participated in experiment 1, 16 healthy individuals (six women) aged 20-29 (mean, 23.8 years) participated in experiment 2 , and 16 healthy individuals ( 10 women) aged 19-33 (mean, 23.3 years) participated in experiment 3. None of the participants took part in more than one experiment. All participants were right-handed according to a standard handedness inventory (Briggs and Nebes, 1975), were native Italian speakers with normal or corrected-to-normal visual acuity in both eyes, and were naive as to the purposes of the experiment.

Information about the experimental hypothesis was provided only after the experimental tests were completed. Participants gave their written informed consent and were paid $15 €$ for their participation in the study. The procedures were approved by the local ethics committee and were in accordance with the ethical standards of the 1964 Declaration of Helsinki. None of the participants had neurological, psychiatric, or other medical problems or any contraindication to TMS (Wasserman, 1998). No discomfort or adverse effects during TMS were reported or noticed.

Electromyography recording and transcranial magnetic stimulation. Motor evoked potentials (MEPs) were recorded simultaneously from the first dorsal interosseous (FDI) and abductor digiti minimi (ADM) muscles (experiments 1 and 2) and from the FDI and the extensor carpi radialis (ECR) muscle (experiment 3 ) of the right hand. It is worth noting here that the FDI muscle is strongly involved in the execution of pincer grips; in contrast, the ADM muscle does not play a major role in the execution of pincer grips but is involved in the execution of gross grasping movements. The ECR muscle does not have any specific role in the execution of either gross or precision grasping movements. Electromyographic (EMG) recordings were performed through surface $\mathrm{Ag} / \mathrm{AgCl}$ cup electrodes ( $1 \mathrm{~cm}$ diameter) placed in a belly tendon montage. Responses were amplified, bandpass filtered $(20 \mathrm{~Hz}$ to $3 \mathrm{kHz}$ ) and digitized by means of a Viking IV electromyography equipment (Nicolet Biomedical, Madison, WI). The sampling rate of the EMG signal was $20 \mathrm{kHz}$. A prestimulus recording of $20 \mathrm{~ms}$ was used to check for the presence of EMG activity before the TMS pulse. To further control for the presence of unwanted background EMG activity before the magnetic pulse, the signal from both muscles was additionally displayed in separate channels set at high sensitivity $(50 \mu \mathrm{V})$. Moreover, during the preliminary session, EMG signals were sent to loudspeakers to provide participants with an auditory feedback of their muscle relaxation.

Focal TMS was performed by means of a $70 \mathrm{~mm}$ figure-eight stimulation coil (Magstim polyurethane-coated coil) connected to a Magstim 200 Rapid (The Magstim Company, Carmarthenshire, Wales, UK), producing a maximum output of $2 \mathrm{~T}$ at the coil surface (pulse duration, 250 $\mu \mathrm{s}$; rise time, $60 \mu \mathrm{s}$ ). The coil was placed tangentially on the scalp, with the handle pointing backward and laterally $45^{\circ}$ away from the midline, approximately perpendicular to the line of the central sulcus. This orientation induced a posterior-anterior current in the brain, which tends to activate corticospinal neurons indirectly via excitatory synaptic inputs (Di Lazzaro et al., 1998). We chose it based on the finding that the lowest motor threshold is achieved when the induced electric current in the brain is flowing approximately perpendicular to the central sulcus (Brasil-Neto et al., 1992; Mills et al., 1992).

During the recording session, the coil was positioned over the left motor cortex in correspondence with the optimal scalp position (OSP), defined as the position from which MEPs with maximal amplitude were recorded. The OSP was detected by moving the intersection of the coil in $1 \mathrm{~cm}$ steps around the motor hand area of the left motor cortex and by delivering TMS pulses with constant intensity. Participants wore a tightly fitting bathing cap on which the scalp positions for stimulation were marked. The coil was held by hand, and its position, with respect to the marks, was checked continuously. We chose to hold the coil by hand to easily compensate for small movements of the participant's head during data collection. Indeed, it has been demonstrated that holding the coil by hand or by a holder induces a comparable MEP variability (Ellaway et al., 1998). Moreover, although image-guided TMS allows for higher resolution of the targeted sites with respect to holding the coil by hand, the two procedures brought about a comparable degree of MEP variability when high, suprathreshold stimulation intensities were used (Gugino et al., 2001). The resting motor threshold (rMT), defined as the lowest stimulus intensity able to evoke five of 10 MEPs with an amplitude of at least 50 $\mu \mathrm{V}$, was determined by holding the stimulation coil over the OSP. Because MEPs were simultaneously recorded from two muscles in all experiments, OSP and rMT were determined by using the higher threshold muscle, namely, the ADM, in experiments 1 and 2, and the ECR in experiment 3. Thus, in each experiment, stimulation conditions were optimal for the muscle that is not activated during actual execution of the observed implied actions. With this procedure, a clear and stable signal was obtained from both targeted muscles in all participants in all experiments. Indeed, the distance between the OSPs for the targeted muscles was within the spatial resolution of the used coil $(\sim 1 \mathrm{~cm})$ (Krings et al., 1998). To record stable MEPs from the two muscles targeted in the different experiments, stimulation intensity during the recording sessions was $130 \%$ of the rMT and ranged from 50 to $92 \%$ (mean, $67.1 \%$ ) of the maximum stimulator output in experiment 1 , from 55 to $93 \%$ (mean, $68.4 \%$ ) in experiment 2, and from 57 to $79 \%$ (mean, 69.9\%) in experiment 3. MEP peak-to-peak amplitudes (in millivolts) were collected and stored on a computer for off-line analysis.

Stimuli and procedure. Stimuli were color pictures taken with a digital camera and modified by means of the Adobe Photoshop software (Adobe Systems, San Jose, CA). Body stimuli represented the right-hand of a man (26 years) and a woman (29 years) during a pincer grip movement. Presenting two different hand stimuli allowed minimizing habituation and loss of attention. The hands of the two models were presented in an equal number of trials.

In experiment 1 , three types of snapshots depicting different hand motion phases were presented: a still hand laying on a table (still hand), a hand in the middle of the grasping movement (implied motion hand), and the end posture of the grasping movement (Fig. 1, End posture). To rule out that the mere observation of graspable objects may, per se, activate the motor system (Chao and Martin, 2000; Nelissen et al., 2005), none of the action snapshots contained any objects.

Hand stimuli were presented on a uniform background and subtended an $18.53^{\circ} \times 19.31^{\circ}$ region. In experiments 2 and 3, the same still- and moving-hand stimuli of experiment 1 were used. In experiment 2, we assessed the specificity of the implied motion effect found in experiment 1 by presenting pictures of two different exemplars of airplanes. Presenting two different airplane stimuli allowed minimizing habituation and loss of attention (Fig. 2). Each airplane was depicted while still on the ground (still object) or during take off (implied motion object). A background context was included in the images to enhance the difference in the implied motion effect induced by still airplanes and airplanes taking off. Pictures of airplanes subtended an $18.53^{\circ} \times 11.26^{\circ}$ region. In experiment 3 , we tested the selectivity of the implied motion effect by using a different type of object stimuli, namely, pictures of two different flowing waterfalls (implied motion objects). As static control, we presented pictures of the same frozen waterfall (still object). Presenting two different waterfall stimuli allowed minimizing habituation and loss of attention (Fig. 3). One type of waterfall picture subtended an $18.53^{\circ} \times 19.31^{\circ}$ region, the other an $18.53^{\circ} \times 6.98^{\circ}$ region. In experiments 2 and 3 , the presented images corresponded to the factorial combination of stimulus type (body, object) and motion (still, implied motion). For each body or nonbody category, corresponding still and motion stimuli were roughly matched for color, luminance, and viewing perspective.

Stimulus-presentation timing, EMG-recording and TMS triggering, as well as randomization of stimuli in a block, were controlled using E-prime V1.1 software (Psychology Software Tools, Pittsburgh, PA) running on a PC. Stimuli remained on the screen for $1500 \mathrm{~ms}$. On each trial, the magnetic pulse was randomly delivered from 267 to $33 \mathrm{~ms}$ before the offset of the stimulus to avoid any priming effects that could affect MEP size. An $8.8 \mathrm{~s}$ blank screen was presented before the next trial. Therefore, 

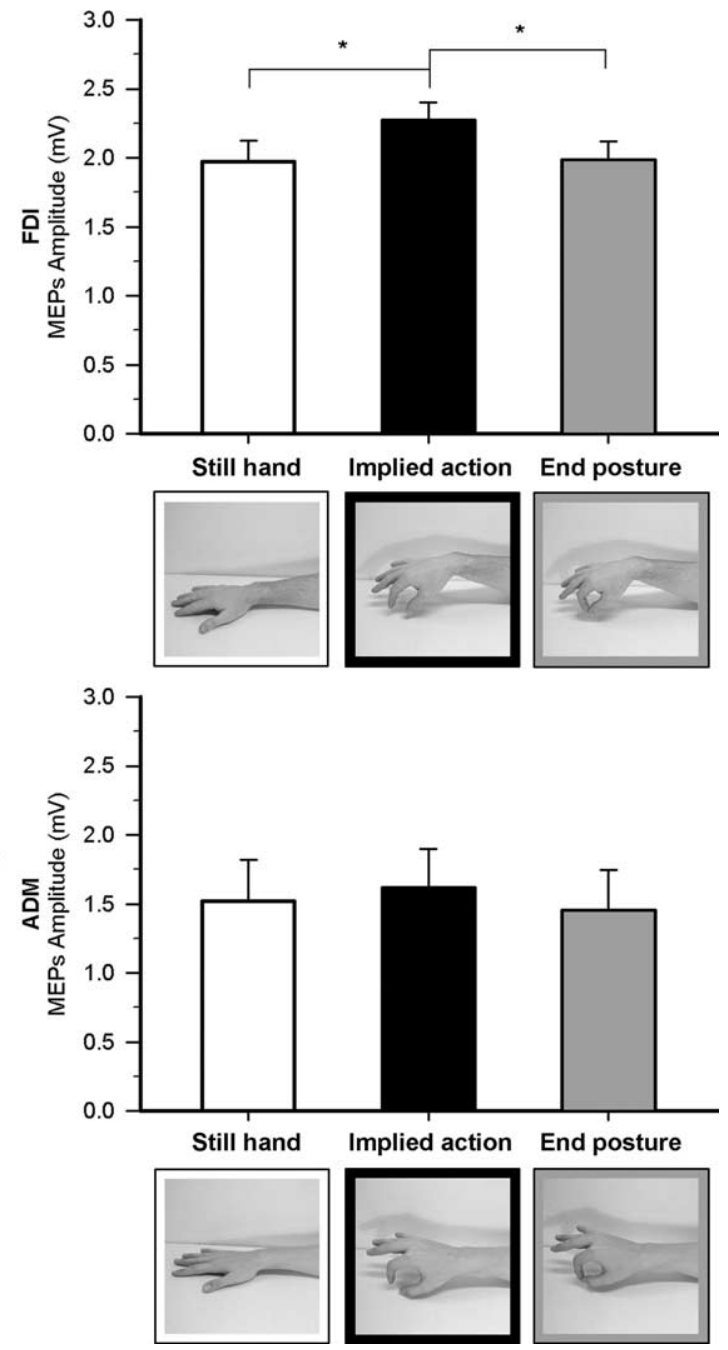

Figure 1. Effect of observation of different implied motion phases of hand action in experiment 1. Raw mean amplitudes (in millivolts) of MEPs recorded from the FDI and ADM muscle during the three observational conditions. The male and female hand stimuli are shown on the FDI and ADM graphs, respectively. Error bars indicate SEs; asterisks indicate significant comparisons.

the interpulse interval ranged from 10.03 to $10.3 \mathrm{~s}$. The choice of the inter-pulse interval was based on research by Chen et al. (1997) that showed no change in corticospinal excitability with repetitive TMS at 0.1 $\mathrm{Hz}$ for $1 \mathrm{~h}$. Participants were tested in one experimental session lasting $\sim 90 \mathrm{~min}$. They sat in a comfortable armchair in a dimly lit room $80 \mathrm{~cm}$ away from a 19 in monitor (resolution, $800 \times 600$ pixels; refresh frequency, $60 \mathrm{~Hz}$ ); they were instructed to keep their right-hand on a pillow and to fully relax their muscles with the help of the auditory feedback coming from the loudspeakers. They were also instructed to pay attention to the stimuli presented on the screen and were informed that at the end of the session questions would be asked about the observed body (gender of the models, orientation of the hand, type of represented action), and nonbody (color and type of airplane and waterfall pictures) stimuli. No overt response was required at any time during stimuli presentation and data collection.

The different observation conditions were presented in separate blocks counterbalanced according to a Latin square design. The order of the different observation blocks did not influence the degree of modulation of MEPs. In experiment 1 , three blocks (still hand, implied motion hand, end posture) were presented for a total of 48 trials; experiments 2 and 3 consisted of four blocks (still and implied motion hand; still and implied motion object) for a total of 64 trials. Each block consisted of 16 trials. A short rest was permitted before proceeding to a different block.
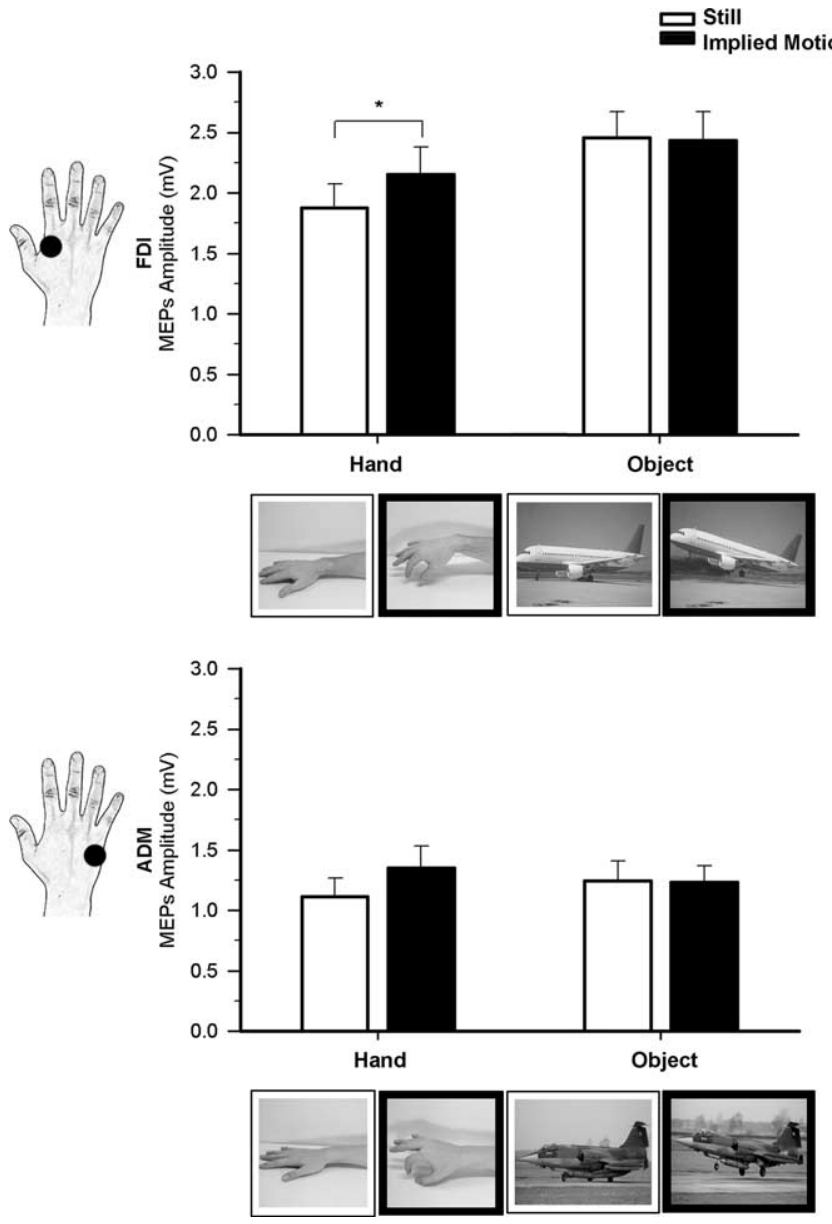

Figure 2. Effect of observation of implied-hand and airplane motion in experiment 2. Raw mean amplitudes (in millivolts) of MEPs recorded from the FDI and ADM muscles during observation of still (white bars) and moving (black bars) hand and object stimuli. The two types of still and moving hands and airplanes are shown on the FDI and ADM graphs respectively. Error bars indicate SEs; asterisks indicate significant comparisons.

Presentation of male and female hands (experiments 1-3) or of the two exemplars of objects (experiments 2 and 3) was randomized. In all experiments, we recorded two series of $8 \mathrm{MEPs}$ while participants kept their eyes closed. One series was recorded at the beginning and the other at the end of the experimental session. Comparisons of MEP amplitudes in these two series allowed us to check for any corticospinal excitability change related to TMS per se.

After the TMS session, printed versions of the experimental stimuli were presented on separate A4 pages in a counterbalanced order. For each image, participants were asked to judge the perceived intensity of the implied motion by marking a vertical, $10 \mathrm{~cm}$ visual analog scale (VAS) with $0 \mathrm{~cm}$ indicating "no effect" and $10 \mathrm{~cm}$ "maximal effect imaginable".

Data handling. The absence of background EMG activity was confirmed by visual inspection of the data. In each experiment, individual mean peak-to-peak MEP amplitudes were separately calculated for each block ( 16 trials per cell) and each muscle. Trials with background activity preceding the TMS pulse or with an MEP amplitude higher or lower than $2 \mathrm{SD}$ of the mean were discarded $(4.3 \%$ in experiment $1,4.4 \%$ in experiment 2 , and $3.9 \%$ of the total in experiment 3 ). In all experiments, mean values were obtained from at least 15 MEPs per condition.

In experiment 1 we tested whether MEPs recorded from each of the targeted muscles were modulated during observation of hand pictures taken in different phases of pincer grip movements. Mean raw MEP amplitudes for each muscle and for VAS ratings were analyzed by means of a series of one-way repeated-measures ANOVAs with hand motion 
phase (still, implied motion, end posture) as a within-subject variable. In experiments 2 and 3 , we investigated the selectivity of motor facilitation during observation of implied motion stimuli by testing the interaction between the type of stimulus and implied motion. MEPs and VAS data were analyzed by a series of two-way repeated-measures ANOVAs with stimulus type (body, object) and motion (still, implied motion) as within-subject variables. We were interested in the influence of different observational conditions on MEPs recorded from a given muscle and not to differences between the targeted muscles. Therefore, MEP amplitudes recorded from each of the targeted muscles were entered in separate ANOVAs. Post hoc multiple comparisons were performed using the Newman-Keuls test. The two series of MEPs recorded while participants kept their eyes closed at the beginning and at the end of the experimental session served as controls for possible changes of corticospinal excitability caused by TMS per se. Series of paired-sample $t$ tests (two-tailed) were used to compare amplitudes of MEPs recorded from FDI, ADM, and ECR muscles in the conditions run at the beginning and at the end of the three experiments

\section{Results}

Experiment 1

This experiment was aimed at testing whether merely observing snapshots of body images implying motion can act on the observer's motor system. We compared corticospinal excitability during observation of static pictures showing still hands with observation of static images of a hand caught in different phases of the same pincer grip movement. While in the intermediate phase, the hand configuration was appropriate for grasping an object, in the end-posture phase, the thumb and index fingers were in contact, thus making object grasping impossible. Figure 1 shows raw MEP amplitudes recorded from FDI and ADM muscles in the three observational conditions.

Analysis of MEP amplitudes recorded from the FDI during the different observation blocks yielded a significant effect of hand motion phase $\left(F_{(2,30)}=4.13 ; p=0.026\right)$. A Post hoc test showed that MEP amplitude was higher during observation of the implied motion phase $(2.27 \pm 0.13 \mathrm{mV})$ than of the still $(1.97 \pm 0.15$ $\mathrm{mV} ; p=0.043)$ and end posture phases $(1.98 \pm 0.14 \mathrm{mV} ; p=$ $0.021)$, which in turn did not differ from one another $(p=$ $0.932)$. No significant effect of hand motion phase $\left(F_{(2,30)}=1.66\right.$; $p=0.207$ ) was found for MEPs recorded from ADM, a muscle not involved in the actual execution of pincer grip movements.

Mean raw MEP amplitudes during the two blocks with closed eyes run at the beginning and at the end of the experiment were not significantly different for either the FDI $(2.02 \pm 0.21 \mathrm{mV}$ vs $\left.1.93 \pm 0.17 \mathrm{mV} ; t_{(15)}=0.48 ; p=0.639\right)$ or the ADM muscle $\left(1.45 \pm 0.27 \mathrm{mV}\right.$ vs $\left.1.53 \pm 0.29 \mathrm{mV} ; t_{(15)}=-0.59 ; p=0.564\right)$. This indicates that TMS, per se, did not induce any changes in corticospinal excitability in our experimental conditions.

To rule out any effect of observing a still hand, average MEP amplitudes in the eyes-closed conditions recorded at the beginning and at the end of the experimental session were compared with MEP amplitudes in the still-hand condition by means of paired sample $t$ tests. No significant difference was observed for either the FDI $\left(1.96 \pm 0.68 \mathrm{mV}\right.$ vs $1.97 \pm 0.62 \mathrm{mV} ; t_{(15)}=-0.09$; $p=0.93)$ or the ADM muscle $(1.47 \pm 1.08 \mathrm{mV}$ vs $1.52 \pm 1.22$ $\left.\mathrm{mV} ; t_{(15)}=-0.4 ; p=0.692\right)$, thus confirming that the still-hand condition did not alter corticospinal excitability.

Analysis of VAS ratings of the motion implied by each stimulus showed a significant effect of hand motion phase $\left(F_{(2,30)}=\right.$ 41.59; $p<0.001)$ because the subjective perception of implied motion was higher for the implied motion $(6.21 \pm 0.61)$ than for the still hand $(0.47 \pm 0.15 ; p<0.001)$ and end-posture snapshots $(4.3 \pm 0.6 ; p=0.006)$. Implied motion ratings for the end posture were significantly higher than for the still-hand phase $(p<$
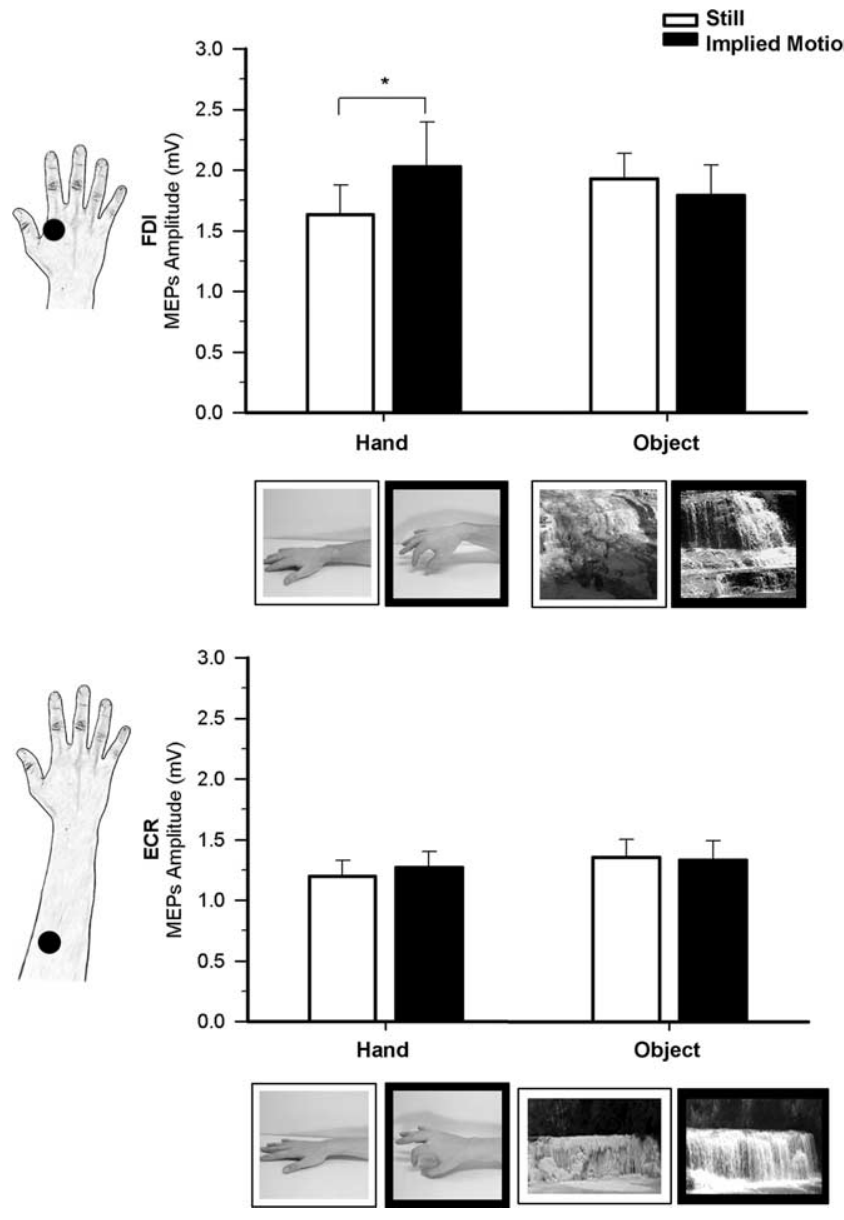

Figure 3. Effect of observation of hands and waterfalls in experiment 3. Raw mean amplitudes (in millivolts) of MEPs recorded from the FDI and ECR muscle during observation of still (white bars) and moving (black bars) hand and object stimuli. The two types of still and moving hand and waterfall stimuli are shown on the FDI and ECR graphs respectively. Error bars indicate SEs; asterisks indicate significant comparisons.

0.001). Thus, observation of the end-posture phase yielded subjective reports of motion information in the absence of motor facilitation. Only observation of the intermediate phase was effective in modulating the excitability of the motor representation of the muscle involved in the execution of the very same movements.

\section{Experiment 2}

This experiment was aimed at testing whether motor facilitation can also occur during observation of implied motion object images. We used snapshots of airplanes that implied or did not imply motion and compared their effects on motor excitability with those of the same hand pictures as in experiment 1. Figure 2 shows raw MEP amplitudes recorded from FDI and ADM muscles in the four observational conditions of experiment 2.

The two-way repeated measures ANOVA performed on MEP amplitudes recorded from the FDI revealed a significant main effect of stimulus type $\left(F_{(1,15)}=16.74 ; p=0.001\right)$, with higher MEP amplitudes during observation of nonbody objects $(2.45 \pm$ $0.22 \mathrm{mV})$ than during observation of body stimuli $(2.01 \pm 0.21$ $\mathrm{mV})$. The main effect of implied motion was marginally significant $\left(F_{(1,15)}=3.55 ; p=0.079\right)$ because mean MEP amplitudes tended to be higher during observation of implied motion stimuli $(2.29 \pm 0.22 \mathrm{mV})$ than during observation of still stimuli $(2.17 \pm$ $0.2 \mathrm{mV}$ ). Crucially, however, a significant stimulus type by mo- 
tion interaction $\left(F_{(1,15)}=4.83 ; p=0.044\right)$ suggested that the effect of implied motion on corticospinal excitability was specific for body stimuli. This was confirmed by post hoc tests showing that MEP amplitude was higher during observation of implied motion hand images $(2.15 \pm 0.23 \mathrm{mV})$ than during observation of still hand images $(1.88 \pm 0.2 \mathrm{mV} ; p=0.012)$. In contrast, no significant difference was observed between implied motion $(2.46 \pm 0.22 \mathrm{mV})$ and still object stimuli $(2.43 \pm 0.24 \mathrm{mV} ; p=$ 0.825). MEP amplitudes during observation of pictures showing still and implied-motion airplanes was significantly higher than during observation of both still hands ( $p<0.001$ for both comparisons) and implied-motion hands ( $p<0.02$ for both comparisons). Therefore, the significant effect of the interaction is explained by the fact that motor facilitation was elicited only by the observation of implied body actions.

The two-way ANOVA performed on MEP amplitudes recorded from the ADM muscle showed that the main effects of stimulus type $\left(F_{(1,15)}<1\right)$ and motion $\left(F_{(1,15)}=2.89 ; p=0.109\right)$ and the stimulus type by motion interaction were nonsignificant $\left(F_{(1,15)}=2.13 ; p=0.165\right)$. Possible inherent differences in excitability of the cortical representations of the FDI and ADM muscles cannot explain the present differential modulation because previous research performed with appropriate experimental conditions has already shown that the responsiveness of the ADM muscle to TMS increases without changes in FDI muscle responsiveness (Romani et al., 2005).

Comparisons of mean raw MEP amplitudes during the eyesclosed condition blocks run at the beginning and the end of the experimental session resulted in nonsignificant differences for both the FDI $\left(1.8 \pm 0.22 \mathrm{mV}\right.$ vs $1.83 \pm 0.18 \mathrm{mV} ; t_{(15)}=-0.19$; $p=0.85)$ and the ADM muscles $(1.02 \pm 0.15 \mathrm{mV}$ vs $1.13 \pm 0.17$ $\left.\mathrm{mV} ; t_{(15)}=-1.26 ; p=0.226\right)$. This indicates that the MEP modulations contingent on action observation found in this experiment are not caused by TMS per se. Moreover, no significant difference was observed between MEPs recorded while participants kept their eyes closed and MEPs recorded during observation of a still hand for either the FDI $(1.8 \pm 0.18 \mathrm{mV}$ vs $1.88 \pm 0.2$ $\left.\mathrm{mV} ; t_{(15)}=-0.62 ; p=0.542\right)$ or the ADM muscle $(1.05 \pm 0.15$ $\mathrm{mV}$ vs $\left.1.11 \pm 0.15 \mathrm{mV} ; t_{(15)}=-0.8 ; p=0.438\right)$.

The two-way repeated-measures ANOVA performed on VAS ratings showed the significance of the main effect of stimulus type $\left(F_{(1,15)}=10.4 ; p=0.006\right)$ because implied motion was higher for nonbody $(4.93 \pm 0.41)$ than for body stimuli $(3.25 \pm 0.29)$. The main effect of motion was highly significant $\left(F_{(1,15)}=64.05 ; p<\right.$ $0.001)$ with higher VAS ratings for implied motion stimuli $(6.46 \pm 0.41)$ than for still stimuli $(1.72 \pm 0.35)$. Importantly, the stimulus type by motion interaction was also significant $\left(F_{(1,15)}=\right.$ $6.15 ; p=0.026)$. Post hoc comparisons showed that mean VAS ratings for implied motion stimuli were significantly higher than for still stimuli in the case of both body $(5.05 \pm 0.44$ vs $1.45 \pm$ $0.45 ; p<0.001)$ and nonbody stimuli $(7.87 \pm 0.62$ vs $2.0 \pm 0.53$; $p<0.001)$. Mean VAS ratings were higher for implied motion airplane stimuli than for implied motion hands $(p<0.001)$. No significant difference was observed between still hands and still airplanes $(p=0.408)$. This pattern of results indicates the following: (1) still hands and airplanes did not evoke perception of implied motion; (2) implied motion of airplanes was perceived as higher than that of hands. Therefore, the body selectivity of the motor facilitation did not reflect differences in the strength of implied motion perception.

\section{Experiment 3}

Results of experiments 1 and 2 indicate that motor facilitation contingent on perception of implied motion is selective for hand stimuli. However, in experiment 2 we found higher corticospinal excitability during observation of still and implied motion airplane pictures as compared with observation of still and implied motion hand pictures. It is known that observation of objects that can be manipulated or grasped activates motor and premotor areas (Chao and Martin, 2000; Beauchamp et al., 2002). Although real airplanes cannot be manipulated, the exemplars presented in our study might have been interpreted as toy models that can be mentally manipulated and grasped. To test this hypothesis we compared motor facilitation contingent on observation of implied body actions with that induced by observation of implied motion of water in waterfall images. Because water is not manipulable, we expected no MEP modulation during observation of the nonbodily object.

Results of the previous two experiments consistently showed that MEPs recorded from the ADM muscle were not modulated during observation of still and implied-motion hands, thus suggesting that motor mapping of implied actions is specific for the cortical representations of the muscles involved in the observed actions. However, one may wonder whether this degree of specificity is limited to intrinsic hand muscles or extends also to other muscles. To address this issue, we further evaluated the muscular selectivity of motor facilitation during observation of implied hand actions by recording MEPs from the ECR (i.e., a forearm muscle not directly involved in the displayed grasping hand actions).

Figure 3 shows raw MEP amplitudes recorded from FDI and ECR muscles in the four observational conditions of experiment 3.

Analysis of MEP amplitudes from the FDI resulted in a nonsignificant main effect of stimulus type $\left(F_{(1,15)}<1\right)$ and motion $\left(F_{(1,15)}=1.43 ; p=0.251\right)$. Crucially, the stimulus type by motion interaction was highly significant $\left(F_{(1,15)}=9.25 ; p=0.008\right)$; a post hoc test showed that mean MEP amplitude was higher during observation of implied-motion hand images $(2.03 \pm 0.37 \mathrm{mV})$ than during observation of still-hand images $(1.64 \pm 0.24 \mathrm{mV}$; $p=0.027)$. In contrast, no significant difference was observed between implied motion $(1.79 \pm 0.25 \mathrm{mV})$ and still object stimuli $(1.93 \pm 0.21 \mathrm{mV} ; p=0.825)$. No other comparisons reached statistical significance (all $p$ values $>0.07$ ), thus showing that the experimental effect was only caused by higher corticospinal excitability during observation of body images with implied motion. No corticospinal facilitation related to object observation was found in experiment 3 , thus ruling out that ceiling effects may have masked modulation of MEP size during observation of still and implied-motion waterfalls. This also suggests that ceiling effects are unlikely to explain the absence of MEP modulation during observation of still and implied-motion airplanes in experiment 2. These results suggest instead that motor mapping of implied actions is specific for body stimuli.

Analysis of MEP amplitude recorded from the ECR muscle showed that neither the main effects of stimulus type $\left(F_{(1,15)}=\right.$ $1.82 ; p=0.198)$ and motion $\left(F_{(1,15)}<1\right)$ nor the stimulus type by motion interaction $\left(F_{(1,15)}<1\right)$ reached significance. The absence of modulation of the ECR muscle supports the notion that motor facilitation during observation of implied body actions is specific for the muscles that are involved in the actual execution of the observed movements. It is worth noting that modulation of the ECR muscle has been obtained during observation of flexionextension movements of the wrist (Borroni et al., 2005); thus, the absence of ECR modulation in the present experiment cannot be 
attributed to a different excitability of the cortical representations of intrinsic hand and forearm muscles.

Comparisons of mean raw MEP amplitudes during the eyesclosed condition blocks run at the beginning and at the end of the experimental session resulted in nonsignificant differences for both the FDI $\left(1.66 \pm 0.22 \mathrm{mV}\right.$ vs $1.46 \pm 0.16 \mathrm{mV} ; t_{(15)}=1.03 ; p=$ $0.32)$ and the ECR muscles $(1.16 \pm 0.12 \mathrm{mV}$ vs $1.19 \pm 0.12 \mathrm{mV}$; $\left.t_{(15)}=-0.47 ; p=0.642\right)$. This indicates that, as in experiments 1 and 2, the MEP modulations contingent on action observation found in this experiment are not caused by TMS per se. No significant difference was observed between MEPs recorded while participants kept their eyes closed and MEPs recorded during observation of a still hand for either the FDI $(1.55 \pm 0.16 \mathrm{mV}$ vs $\left.1.64 \pm 0.24 \mathrm{mV} ; t_{(15)}=-0.40 ; p=0.692\right)$ or the ECR muscle $\left(1.16 \pm 0.12 \mathrm{mV}\right.$ vs $\left.1.2 \pm 0.13 \mathrm{mV} ; t_{(15)}=-0.46 ; p=0.652\right)$, thus showing that also in this experiment observation of a still hand did not alter corticospinal excitability as compared with the eyesclosed condition.

The two-way repeated-measures ANOVA performed on VAS ratings disclosed a significant stimulus type $\left(F_{(1,15)}=12.31 ; p=\right.$ $0.003)$ main effect in that higher implied motion ratings were assigned to nonbody (waterfalls, $4.6 \pm 0.26$ ) than to body stimuli (hands, $3.2 \pm 0.41$ ). The significance of the main effect of motion $\left(F_{(1,15)}=100.58 ; p<0.001\right)$ is attributable to the fact that implied motion stimuli $(6.35 \pm 0.33)$ were rated as more "dynamic" than still stimuli $(1.45 \pm 0.41)$. The stimulus type by motion interaction $\left(F_{(1,15)}=20.61 ; p<0.001\right)$ was also significant. Post hoc comparisons showed the following: the mean VAS rating for implied motion stimuli was significantly higher than for still stimuli for both hands $(4.98 \pm 0.54$ vs $1.42 \pm 0.41 ; p<0.001)$ and waterfalls ( $7.72 \pm 0.32$ vs $1.48 \pm 0.47 ; p<0.001)$. Interestingly, implied-motion waterfalls were considered as more dynamic than implied-motion hands $(p<0.001)$, whereas no significant difference was observed when comparing still waterfalls and still hands ( $p=0.883$ ). Thus, the pattern of implied motion perception for waterfall images was similar to that for airplane images (experiment 2). Crucially, however, no MEP modulation was found during the observation of nongraspable waterfalls either for still images or for implied motion images.

In none of the three experiments did the gender of the model or of the onlooker seem to influence the modulation effects contingent on observation of implied movements of hands or objects.

\section{Discussion}

The present study shows for the first time that mirror motor mapping of actions occurs when dynamic information about body actions is inferred from static pictures of body postures.

\section{Motor mirror mapping of implied body and nonbody motion} Our results indicate that even in the absence of explicit motion of the stimulus, observation of static photographs of pincer grips with implied motion produced a clear increase in corticospinal excitability with respect to observation of static images of still hands. Although entirely novel, this effect resembles that of observing actual actions reported in previous TMS studies of action observation (Fadiga et al., 1995; Strafella and Paus, 2000; Gangitano et al., 2001; Romani et al., 2005). We found that the MEP modulation was selective (i.e., that it was present) only for the FDI muscle that would be activated during actual execution of the observed movement. In contrast, no motor facilitation was observed for ADM and ECR, which have no direct role in the execution of that action. The fact that corticospinal excitability was higher during presentation of still and implied motion airplanes than during presentation of still and implied action (experiment 2) deserves discussion.

On one hand, the comparable MEP amplitude during the viewing of still and implied motion airplanes may indicate the greater likelihood that these pictures will draw attention than hand pictures and, thus, induce general brain activation, including both premotor and motor areas. Ventral premotor cortex activity has been shown to increase during observation of complex nonmanipulable objects (Kellenbach et al., 2003; Nelissen et al., 2005) and abstract visual scenes with dynamic properties (Schubotz and von Cramon, 2002). This explanation, however, does not account for the fact that during the observation of airplane images the MEP amplitude increase was apparent for the FDI muscle, which is active during actual fine grasping, but not for the ADM muscle, which is not. Another possible explanation is that the airplane images were interpreted by the observers as images of toys and, thus, mentally grasped at an implicit level. Indeed, toys are eminently manipulable, and it is known that the observation of toys, tools, and other manipulable objects (Chao and Martin, 2000; Beauchamp et al., 2002), as well as of mimicked manipulation actions (Nelissen et al., 2005), activates premotor cortical areas. Results of experiment 3 also speak in favor of this hypothesis in that no MEP modulation was found during observation of nongraspable objects (waterfalls). Although implied motion was reported for flowing but not for frozen waterfalls, observation of these stimuli produced a comparable pattern of corticospinal excitability. Therefore, the motor facilitation evoked by perception of implied body actions is a specific process that cannot be explained by simple semantic coding of implied motion but seems to be linked to the activation of the frontal node of the mirror network that matches observed and performed actions (Rizzolatti and Craighero, 2004).

This system seems to be important for understanding the goal of an action in that the passive observation of the realized goal of hand-object interactions depicted in static pictures induces a selective activation of the left and right precentral cortex and inferior frontal gyri (Johnson-Frey et al., 2003; Rizzolatti and Craighero, 2004). Moreover, motor and premotor activation was higher during observation of pictures of objects being grasped (goaldirected action) than during observation of the same objects simply being touched (no goal action). This result suggests that mere observation of the goal of the action is enough to trigger mirror motor activation (Johnson-Frey et al., 2003). However, motor and premotor cortex activation in humans is found also during observation of intransitive and apparently aimless body movements (Fadiga et al., 1995; Iacoboni et al., 1999; Buccino et al., 2001; Costantini et al., 2005; Romani et al., 2005). Here, we demonstrate that the neural observation-execution matching system can be recruited by merely extracting motion information implied by a static body.

\section{Motor facilitation and anticipatory mental simulation of the observed action}

Behavioral studies indicate that an observer's actual movement can be facilitated by viewing congruent compared with incongruent movements performed by others (Brass et al., 2000, 2001; Sturmer et al., 2000; Kilner et al., 2003), or even by single static frames suggesting these congruent or incongruent movements (Brass et al., 2000; Craighero et al., 2002; Vogt et al., 2003). Static pictures of moving biological or nonbiological objects typically convey dynamic information concerning the position of an object just before and after the picture was taken. Therefore, pro- 
cessing implied motion is inherently temporal. Another important result of the present study is that facilitation of the FDI muscle response was present during observation of the static image that was most suggestive of a hand caught in action, whereas it was absent not only during observation of a resting, relaxed hand but also during observation of a hand image suggesting a completed action. Although the implied motion of the latter image was subjectively rated as higher than that of the resting hand, this was apparently insufficient to engage the motor system. This dissociation between subjective reports and MEP modulation indicates that semantic representation of the movement cannot be the sole explanation of the motor facilitation contingent on observation of implied actions. One likely account for the different motor modulation induced by the two types of implied-motion hand stimuli is that the one caught in action conveyed dynamic information about forward and backward action paths, whereas the final posture hand provided information only about backward action paths. This would suggest that the motor system was maximally activated by the extrapolation of the future trajectory of body actions. This is in keeping with the forward bias in recognition memory observed in the representational-momentum paradigm (Freyd, 1983; Verfaillie and Daems, 2002). The anticipatory representation of motion may be related to the functional significance of implied motion perception. Indeed, although the full sequence of motion is rarely visible during interactions with a dynamic world, anticipation of the future position of moving entities may allow us to bridge discontinuities in visual inputs and, thus, to interact optimally with the external world.

The extrapolation of motion information from static pictures and the forward distortion of the movement of a target object (representational momentum) rely on a large neural network that includes higher-order prefrontal and parietal areas (Amorim et al., 2000; Rao et al., 2004) but also higher-order visual areas such as the MT/MST complex (Kourtzi and Kanwisher, 2000; Senior et al., 2000; Lorteije et al., 2006). Studies in humans (Krekelberg et al., 2005) and monkeys (Krekelberg et al., 2003) show that the same populations of cells in extrastriate visual areas code for both implied and real motion. Moreover, MT is activated during mental imagery and perception of actual and implied motion (Slotnick et al., 2005). It is possible that mental imagery of forward motion paths (Munger et al., 1999) allows us to fill in the missing visual information and thus to create representational momentum effects.

Previous research about the neural underpinnings of impliedmotion perception did not distinguish between biological and nonbiological motion. However, studies indicate that observation and imagery of human body movements not only activate visual areas but also neural structures typically involved in motor planning and execution of the very same actions (Porro et al., 1996; Fadiga et al., 1999; Iacoboni et al., 1999; Fourkas et al., 2006) that are part of the so-called mirror neuron system (Rizzolatti and Craighero, 2004). Mirror neurons respond to either selfproduced or observed actions. Importantly, these neurons continue to respond also when the final phase of the action is occluded from sight but can be guessed by the observing monkey (Umiltà et al., 2001). Moreover, motor activation in humans has been found in response to symbolic cues signaling an upcoming movement, thus suggesting a role for the mirror system in predicting and anticipating the actions of other individuals (Kilner et al., 2004). It is also important that activation of the corticospinal motor system contingent on actual observation of grasping movements was modulated by the temporal progress of the observed hand action, with higher motor facilitation during the opening phase (Gangitano et al., 2001). Moreover, the artificial introduction of delayed aperture or sudden closure of fingers suppressed the facilitation of the motor cortex, thus suggesting that mirror mapping is affected by the predictability of the sequence of observed movements (Gangitano et al., 2004). A clear temporal modulation of amplitude of MEPs recorded from two hand muscles (flexor digitorum superficialis and FDI) was also found during observation of videos showing a hand reaching, grasping, and holding a sphere (Montagna et al., 2005). It is relevant that FDI facilitation was found not only during observation of the finger closing phase but also during observation of hand closure and active object holding phase (i.e., when the action was still ongoing). Our study extends this result by suggesting that the FDI facilitation triggered by implied motion occurs when observing ongoing but not yet completed body actions.

\section{References}

Amorim MA, Lang W, Lindinger G, Mayer D, Deecke L, Berthoz A (2000) Modulation of spatial orientation processing by mental imagery instructions: a MEG study of representational momentum. J Cogn Neurosci $12: 569-582$.

Beauchamp MS, Lee KE, Haxby JV, Martin A (2002) Parallel visual motion processing streams for manipulable objects and human movements. Neuron 34:149-159.

Borroni P, Montagna M, Cerri G, Baldissera F (2005) Cyclic time course of motor excitability modulation during the observation of a cyclic hand movement. Brain Res 1065:115-124.

Brasil-Neto JP, Cohen LG, Panizza M, Nilsson J, Roth BJ, Hallett M (1992) Optimal focal transcranial magnetic activation of the human motor cortex: effects of coil orientation, shape of the induced current pulse, and stimulus intensity. J Clin Neurophysiol 9:132-136.

Brass M, Bekkering H, Wohlschlager A, Prinz W (2000) Compatibility between observed and executed finger movements: comparing symbolic, spatial, and imitative cues. Brain Cogn 44:124-143.

Brass M, Bekkering H, Prinz W (2001) Movement observation affects movement execution in a simple response task. Acta Psychol (Amst) 106:3-22.

Briggs GG, Nebes RD (1975) Patterns of hand preference in a student population. Cortex 11:230-238.

Buccino G, Binkofski F, Fink GR, Fadiga L, Fogassi L, Gallese V, Seitz RJ, Zilles K, Rizzolatti G, Freund HJ (2001) Action observation activates premotor and parietal areas in a somatotopic manner: an fMRI study. Eur J Neurosci 13:400-404.

Chao LL, Martin A (2000) Representation of manipulable man-made objects in the dorsal stream. NeuroImage 12:478-484.

Chen R, Classen J, Gerloff C, Celnik P, Wassermann EM, Hallett M, Cohen LG (1997) Depression of motor cortex excitability by low-frequency transcranial magnetic stimulation. Neurology 48:1398-1403.

Costantini M, Galati G, Ferretti A, Caulo M, Tartaro A, Romani GL, Aglioti SM (2005) Neural systems underlying observation of humanly impossible movements: an fMRI study. Cereb Cortex 15:1761-1767.

Craighero L, Bello A, Fadiga L, Rizzolatti G (2002) Hand action preparation influences the responses to hand pictures. Neuropsychologia 40:492-502.

Di Lazzaro V, Oliviero A, Profice P, Saturno E, Pilato F, Insola A, Mazzone P, Tonali P, Rothwell JC (1998) Comparison of descending volleys evoked by transcranial magnetic and electric stimulation in conscious humans. Electroencephalogr Clin Neurophysiol 109:397-401.

Dupont P, Orban GA, De Bruyn B, Verbruggen A, Mortelmans L (1994) Many areas in the human brain respond to visual motion. J Neurophysiol 72:1420-1424.

Ellaway PH, Davey NJ, Maskill DW, Rawlinson SR, Lewis HS, Anissimova NP (1998) Variability in the amplitude of skeletal muscle responses to magnetic stimulation of the motor cortex in man. Electroencephalogr Clin Neurophysiol 109:104-113.

Fadiga L, Fogassi L, Pavesi G, Rizzolatti G (1995) Motor facilitation during action observation: a magnetic stimulation study. J Neurophysiol 73:2608-2611.

Fadiga L, Buccino G, Craighero L, Fogassi L, Gallese V, Pavesi G (1999) Corticospinal excitability is specifically modulated by motor imagery: a magnetic stimulation study. Neuropsychologia 37:147-158. 
Fadiga L, Craighero L, Olivier E (2005) Human motor cortex excitability during the perception of others' action. Curr Opin Neurobiol 15:213-218.

Finke RA, Freyd JJ (1985) Transformations of visual memory induced by implied motions of pattern elements. J Exp Psychol Learn Mem Cogn 11:780-794.

Finke RA, Shyi GC (1988) Mental extrapolation and representational momentum for complex implied motions. J Exp Psychol Learn Mem Cogn $14: 112-120$.

Fourkas AD, Avenanti A, Urgesi C, Aglioti SM (2006) Corticospinal facilitation during first and third person imagery. Exp Brain Res 168:143-151.

Freyd JJ (1983) The mental representation of movement when static stimuli are viewed. Percept Psychophys 33:575-581.

Gangitano M, Mottaghy FM, Pascual-Leone A (2001) Phase-specific modulation of cortical motor output during movement observation. NeuroReport 12:1489-1492.

Gangitano M, Mottaghy FM, Pascual-Leone A (2004) Modulation of premotor mirror neuron activity during observation of unpredictable grasping movements. Eur J Neurosci 20:2193-2202.

Grossman E, Donnelly M, Price R, Pickens D, Morgan V, Neighbor G, Blake $\mathrm{R}$ (2000) Brain areas involved in perception of biological motion. J Cogn Neurosci 12:711-720.

Gugino LD, Romero JR, Aglio L, Titone D, Ramirez M, Pascual-Leone A, Grimson E, Weisenfeld N, Kikinis R, Shenton ME (2001) Transcranial magnetic stimulation coregistered with MRI: a comparison of a guided versus blind stimulation technique and its effect on evoked compound muscle action potentials. Clin Neurophysiol 112:1781-1792.

Hermsdörfer J, Goldenberg G, Wachsmuth C, Conrad B, Ceballos-Baumann AO, Bartenstein P, Schwaiger M, Boecker H (2001) Cortical correlates of gesture processing: clues to the cerebral mechanisms underlying apraxia during the imitation of meaningless gestures. NeuroImage 14:149-161.

Iacoboni M, Woods RP, Brass M, Bekkering H, Mazziotta JC, Rizzolatti G (1999) Cortical mechanisms of human imitation. Science 286:2526-2528.

Jellema T, Perrett DI (2003) Cells in monkey STS responsive to articulated body motions and consequent static posture: a case of implied motion? Neuropsychologia 41:1728-1737.

Johnson-Frey SH, Maloof FR, Newman-Norlund R, Farrer C, Inati S, Grafton ST (2003) Actions or hand-object interactions? Human inferior frontal cortex and action observation. Neuron 39:1053-1058.

Kellenbach ML, Brett M, Patterson K (2003) Actions speak louder than functions: the importance of manipulability and action in tool representation. J Cogn Neurosci 15:30-46.

Kilner JM, Paulignan Y, Blakemore SJ (2003) An interference effect of observed biological movement on action. Curr Biol 13:522-525.

Kilner JM, Vargas C, Duval S, Blakemore SJ, Sirigu A (2004) Motor activation prior to observation of a predicted movement. Nat Neurosci 7:1299-1301.

Kourtzi Z, Kanwisher N (2000) Activation in human MT/MST by static images with implied motion. J Cogn Neurosci 12:48-55.

Krekelberg B, Dannenberg S, Hoffmann KP, Bremmer F, Ross J (2003) Neural correlates of implied motion. Nature 424:674-677.

Krekelberg B, Vatakis A, Kourtzi Z (2005) Implied motion from form in the human visual cortex. J Neurophysiol 94:4373-4386.

Krings T, Naujokat C, von Keyserlingk DG (1998) Representation of cortical motor function as revealed by stereotactic transcranial magnetic stimulation. Electroencephalogr Clin Neurophysiol 109:85-93.

Lorteije JA, Kenemans JL, Jellema T, van der Lubbe RH, de Heer F, van Wezel RJ (2006) Delayed response to animate implied motion in human motion processing areas. J Cogn Neurosci 18:158-168.

Mills KR, Boniface SJ, Schubert M (1992) Magnetic brain stimulation with a double coil: the importance of coil orientation. Electroencephalogr Clin Neurophysiol 85:17-21.
Montagna M, Cerri G, Borroni P, Baldissera F (2005) Excitability changes in human corticospinal projections to muscles moving hand and fingers while viewing a reaching and grasping action. Eur J Neurosci 22:1513-1520.

Munger MP, Solberg JL, Horrocks KK (1999) The relationship between mental rotation and representational momentum. J Exp Psychol Learn Mem Cogn 25:1557-1568.

Nelissen K, Luppino G, Vanduffel W, Rizzolatti G, Orban GA (2005) Observing others: multiple action representation in the frontal lobe. Science 310:332-336.

Peigneux P, Salmon E, van der Linden M, Garraux G, Aerts J, Delfiore G, Degueldre C, Luxen A, Orban G, Franck G (2000) The role of lateral occipitotemporal junction and area MT/V5 in the visual analysis of upper-limb postures. NeuroImage 11:644-655.

Peuskens H, Vanrie J, Verfaillie K, Orban GA (2005) Specificity of regions processing biological motion. Eur J Neurosci 21:2864-2875.

Porro CA, Francescato MP, Cettolo V, Diamond ME, Baraldi P, Zuiani C, Bazzocchi M, di Prampero PE (1996) Primary motor and sensory cortex activation during motor performance and motor imagery: a functional magnetic resonance imaging study. J Neurosci 16:7688-7698.

Puce A, Perrett D (2003) Electrophysiology and brain imaging of biological motion. Philos Trans R Soc Lond B Biol Sci 358:435-445.

Rao H, Han S, Jiang Y, Xue Y, Gu H, Cui Y, Gao D (2004) Engagement of the prefrontal cortex in representational momentum: an fMRI study. NeuroImage 23:98-103.

Rizzolatti G, Craighero L (2004) The mirror-neuron system. Annu Rev Neurosci 27:169-192.

Romani M, Cesari P, Urgesi C, Facchini S, Aglioti SM (2005) Motor facilitation of the human cortico-spinal system during observation of biomechanically impossible movements. NeuroImage 26:755-763.

Schubotz RI, von Cramon DY (2002) A blueprint for target motion: fMRI reveals perceived sequential complexity to modulate premotor cortex. NeuroImage 16:920-935.

Senior C, Barnes J, Giampietro V, Simmons A, Bullmore ET, Brammer M, David AS (2000) The functional neuroanatomy of implicit-motion perception or representational momentum. Curr Biol 10:16-22.

Slotnick SD, Thompson WL, Kosslyn SM (2005) Visual mental imagery induces retinotopically organized activation of early visual areas. Cereb Cortex 15:1570-1583.

Strafella AP, Paus T (2000) Modulation of cortical excitability during action observation: a transcranial magnetic stimulation study. NeuroReport 11:2289-2292.

Sturmer B, Aschersleben G, Prinz W (2000) Correspondence effects with manual gestures and postures: a study of imitation. J Exp Psychol Hum Percept Perform 26:1746-1759.

Tootell RB, Reppas JB, Kwong KK, Malach R, Born RT, Brady TJ, Rosen BR, Belliveau JW (1995) Functional analysis of human MT and related visual cortical areas using magnetic resonance imaging. J Neurosci 15:3215-3230.

Umiltà MA, Kohler E, Gallese V, Fogassi L, Fadiga L, Keysers C, Rizzolatti G (2001) I know what you are doing: a neurophysiological study. Neuron 31:155-165.

Verfaillie K, Daems A (2002) Representing and anticipating human actions in vision. Vis Cogn 9:217-232.

Vogt S, Taylor P, Hopkins B (2003) Visuomotor priming by pictures of hand postures: perspective matters. Neuropsychologia 41:941-951.

Wasserman EM (1998) Risk and safety of repetitive transcranial magnetic stimulation: report and suggested guidelines from the International Workshop on the Safety of Repetitive Transcranial Magnetic Stimulation, June 5-7, 1996. Electroencephalogr Clin Neurophysiol 108:1-16.

Zeki S, Watson JD, Lueck CJ, Friston KJ, Kennard C, Frackowiak RS (1991) A direct demonstration of functional specialization in human visual cortex. J Neurosci 11:641-649. 